\title{
Stability of microtubule attachment to metaphase kinetochores in $\mathrm{PtK}_{1}$ cells
}

\author{
LYNNE CASSIMERIS ${ }^{1, *}$, CONLY L. RIEDER ${ }^{2,3}$, GERALD RUPP ${ }^{2, \dagger}$ and E. D. SALMON ${ }^{1}$ \\ ${ }^{1}$ Department of Biology, CB\#3280, University of North Carolina, Chapel Hill, NC 27599-3280, USA \\ ${ }^{2}$ Wadsworth Center for Laboratories and Research, New York State Department of Health, Albany, NY 12201-0509, USA and \\ ${ }^{3}$ School of Public Health, SUNY-Albany, Albany, NY 12222, USA
}

* Author for correspondence at: Department of Biology, University of Pennsylvania, Philadelphia, PA 19104, USA

$\dagger$ Present address: Department of Anatomy and Cell Biology, SUNY-Buffalo, Buffalo, NY 14214, USA

\section{Summary}

Kinetochore microtubules are known to be differentially stable to a variety of microtubule depolymerization agents compared to the non-kinetochore polar microtubules, but the dynamics of microtubule attachment to the kinetochore is currently controversial. We have examined the stability of kinetochore microtubules in metaphase $\mathrm{PtK}_{1}$ spindles at $23^{\circ} \mathrm{C}$ when microtubule assembly is abruptly blocked with the drug nocodazole. Metaphase cells were incubated in medium containing $34 \mu \mathrm{M}$ nocodazole for various times before fixation and processing either for immunofluorescence light microscopy or serial-section electron microscopy. Microtubules not associated with kinetochore fibers disappeared completely in less than $1 \mathrm{~min}$. Kinetochore fibers persisted and shortened, as the spindle poles moved close to the chromosomes over a 10-20 min interval. During this shortening process, the number of kin- etochore microtubules decreased slowly. The mean number of kinetochore microtubules was $24 \pm 5$ in control cells and zero in cells incubated with nocodazole for $20 \mathrm{~min}$. The half-time of microtubule attachment to the kinetochore was $\sim 7.5 \mathrm{~min}$. These results show that when microtubule assembly is blocked, kinetochore microtubules shorten more slowly and persist about 10 times longer than the labile polar microtubules. If kinetochore microtubules shorten by tubulin dissociation at their plus-ends like the non-kinetochore polar microtubules, then the microtubule surface lattice must be able to translocate through the kinetochore attachment site without frequent detachment occurring.

Key words: metaphase, kinetochore, microtubule.

\section{Introduction}

Chromosomes are segregated during mitosis by interactions between their kinetochores and the plusends of spindle microtubules (Nicklas, 1987; Mitchison, 1988; Salmon, 1989). Initially, a kinetochore on a chromosome attaches to polar microtubules from one spindle pole. When the opposing kinetochore attaches to microtubules from the opposite pole, the chromosome moves to the spindle equator, a position equidistant between the poles. This movement requires the concurrent lengthening of one kinetochore fiber and the shortening of the opposite fiber. At anaphase onset the sister chromatids separate and their kinetochore fibers shorten as the chromatids move poleward (reviewed by Mitchison, 1988; McIntosh and Koonce, 1989; Salmon, 1989).

Recent experiments have determined that labeled tubulin subunits add onto kinetochore microtubules at a site proximal to the kinetochore (Mitchison et al. 1986; Mitchison, 1988; Geuens et al. 1989). One interpretation of these results is that lengthening of a kinetochore fiber occurs by the growth and translocation of individual kinetochore microtubules at their attachment sites (Mitchison, 1988). Alternatively, the presence of kinetochore microtubules with labeled subunits localized proximal to the kinetochore could arise if the kinetochores rapidly released and rebound microtubules (free microtubule plus-ends would

Journal of Cell Science 96, 9-15 (1990)

Printed in Great Britain (C) The Company of Biologists Limited 1990 elongate, incorporating labeled subunits and subsequent capture by the kinetochore would result in labeled subunits localized proximal to the kinetochore) (Cassimeris et al. 1988; Salmon, 1989).

In this paper we have investigated this issue by examining the stability of kinetochore microtubules at metaphase by blocking microtubule assembly. Microtubule elongation can be selectively inhibited in reconstituted preparations in vitro by simply diluting the tubulin concentration (Koshland et al. 1988). In living-cell experiments, selective dilution of the tubulin concentration has not been possible. However, a complex of tubulin and a colchicine-like drug can be used to block microtubule assembly rapidly in vivo (Salmon et al. 1984; Cassimeris et al. 1986). In the experiments reported here, we treated metaphase $\mathrm{PtK}_{1}$ cells at room temperature with high concentrations ( $34 \mu \mathrm{M})$ of the tubulin-binding drug nocodazole and monitored the disappearance and shortening of kinetochore microtubules by immunofluorescence and serial-section electron microscopy. This concentration of nocodazole has been shown to induce rapid disappearance (half-life about $10 \mathrm{~s}$ at $37^{\circ} \mathrm{C}$ in $\mathrm{PtK}_{1}$ cells (Salmon et al. 1984; Salmon, 1989)) of the non-kinetochore polar microtubules. Nocodazole rapidly penetrates the plasma membrane and has been shown to bind to the colchicine binding site on the tubulin dimer; this site is not exposed when the dimer is incorporated into the lattice wall of a microtubule 
(Margolis and Wilson, 1977; Hoebeke et al. 1976; Lee et al. 1980). Our study was done at room temperature in order to correlate results with data on spindle microtubule dynamics we measured previously, using fluorescently labeled tubulin, and measurements of fluorescence redistribution after photobleaching (FRAP) (Cassimeris et al. 1988).

\section{Materials and mothods}

\section{Cell culture and nocodazole incubation}

Stock cultures of $\mathrm{PtK}_{1}$ cells were maintained at $37^{\circ} \mathrm{C}$ in Ham's F-10 medium supplemented with fetal bovine serum and antibiotics. Cells were plated onto polylysine-coated coverslips and used within 2 days after subculture. For experiments cells were transferred to $23^{\circ} \mathrm{C}$ Hepes-buffered culture medium and allowed to equilibrate at this temperature for 20-25 min. Coverslips were transferred to Hepes-buffered culture medium containing $10 \mu \mathrm{g} \mathrm{ml}^{-1}$ (34 $\left.\mu \mathrm{M}\right)$ nocodazole (Sigma) and fixed after various times in nocodazole.

At $23^{\circ} \mathrm{C} \mathrm{PtK}_{1}$ cells proceed through mitosis, but at a much slower rate than at $37^{\circ} \mathrm{C}$ (Rieder, 1981b). As a result of this extended time in mitosis several metaphase cells could be marked on each coverslip using a circular diamond objective scribe prior to incubation in nocodazole.

\section{Indirect immunofluorescence}

Coverslips of control and nocodazole-treated cells were fixed and processed for anti-tubulin immunofluorescence as described previously (Cassimeris et al. 1986). After staining, cells were examined and photographed with a Nikon $100 \times / 1.4$ NA Plan Apochromat objective mounted on a Zeiss Universal microscope stand.

\section{Electron microscopy}

Coverlips of control and nocodazole-treated cells were fixed for $30 \mathrm{~min}$ at $23^{\circ} \mathrm{C}$ in $3 \%$ glutaraldehyde in $0.1 \mathrm{M}$ cacodylate buffer (pH 7.1). They were then washed in buffer, postfixed in $1 \% \mathrm{OsO}_{4}$ (in cacodylate buffer) for $15 \mathrm{~min}$ at $4^{\circ} \mathrm{C}$, dehydrated in a graded series of ethanols, and flat embedded in Epon/Araldite. One to two cells from each time point, which were marked prior to nocodazole incubation, were relocated within each embedment, excised, and mounted on Epon pegs. Each cell was then serially thin eectioned with a diamond knife using a Sorvall Mt 6000 ultramicrotome. Ribbons of serial sections were mounted on Formvar-coated slot grids and stained with uranyl acetate and lead citrate (Rieder, 1981a). Sections were examined and photographed with either a Philips 301 or a Zeiss EM10C electron microscope operated at $80 \mathrm{kV}$.

\section{Data analysis}

Electron microscopic negatives of serial sections were printed to a final magnification of $\times 20000$ to $\times 50000$. The number of microtubules terminating within the kinetochore (i.e. kinetochore microtubules) was then determined from the serial photographic prints. The kinetochore was defined on the basis of its trilaminar appearance in thin-section electron micrographs. Microtubules that clearly terminated within this recognizable structure were defined as kinetochore microtubules. In most cases, the entire kinetochore region was contained within six serial thin sections.

\section{Results}

Kinetochore fibers are distinct in immunofluorescence micrographs of metaphase spindles fixed before nocodazole addition, but predominant in spindles fixed $10 \mathrm{~s}$ (data not shown) or longer after nocodazole addition (Fig. 1). Few, or no, non-kinetochore fiber microtubules were seen in cells fixed after $\sim 30-60 \mathrm{~s}$ in nocodazole and no microtubules of any kind were seen in cells fixed $\sim 20$ min after incubation
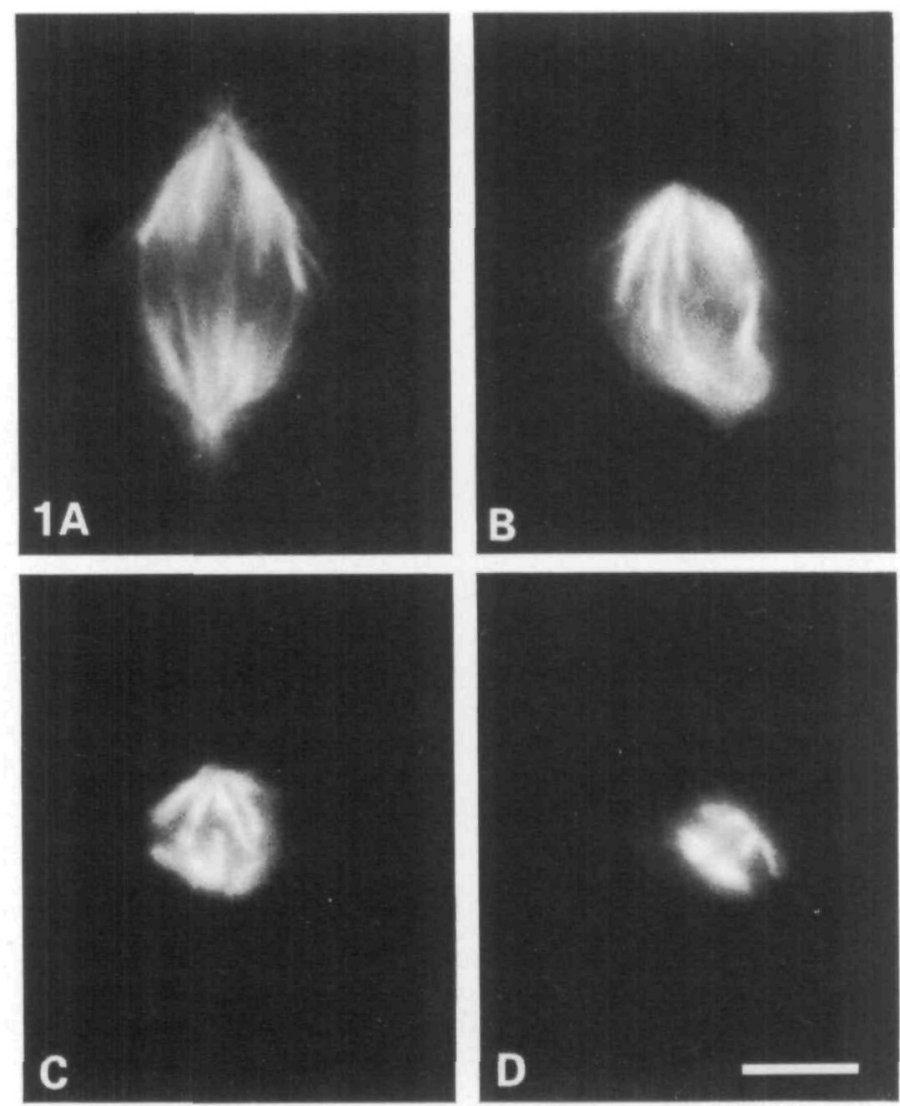

Fig. 1. Immunofluorescence micrographs of metaphase $\mathrm{PtK}_{1}$ cells fixed after incubation in nocodazole. A. Control; B, 3 min; C, $5 \mathrm{~min}$; and D, $10 \mathrm{~min}$ incubation in nocodazole. Bar, $5 \mu \mathrm{m}$.

in nocodazole. Bundles of kinetochore fiber microtubules were seen to span the distance between the kinetochores and the poles in electron micrographs of cells incubated with nocodazole for less than $20 \mathrm{~min}$. In favorable sections, (those cut parallel to a kinetochore fiber), many microtubules could be followed continuously from the kinetochore to the pole (data not shown, similar to those of Rieder, 1981a and Witt et al. 1981).

The differentially stable kinetochore fibers shortened considerably when microtubule assembly was blocked and by $10 \mathrm{~min}$ in nocodazole they were only $\sim 25-45 \%$ of their initial length. The poles moved inward to the chromosomes as the kinetochore fibers shortened. The kinetochore fibers always appeared to extend from the poles to the primary constrictions of the chromosomes; they never appeared to be detached from either the kinetochores or the poles in either fluorescent (Fig. 1) or electron micrographs (data not shown) of cells fixed during the shortening process. This result confirms previous observations of kinetochore fiber shortening after treatment of spindles with colchicine-like drugs, high hydrostatic pressure or cooling (reviewed by Inoue, 1981; Salmon, 1989).

The number of microtubules attached to a kinetochore decreased slowly with longer incubations in nocodazole, as seen in the electron micrographs in Figs 2 and 3. Representative sections through kinetochores at each time point are shown in Fig. 2. Fig. 3 shows serial sections through kinetochores fixed at $3 \mathrm{~min}, 5 \mathrm{~min}$ and $20 \mathrm{~min}$ after incubation in nocodazole. We determined the mean number of kinetochore microtubules and the average 

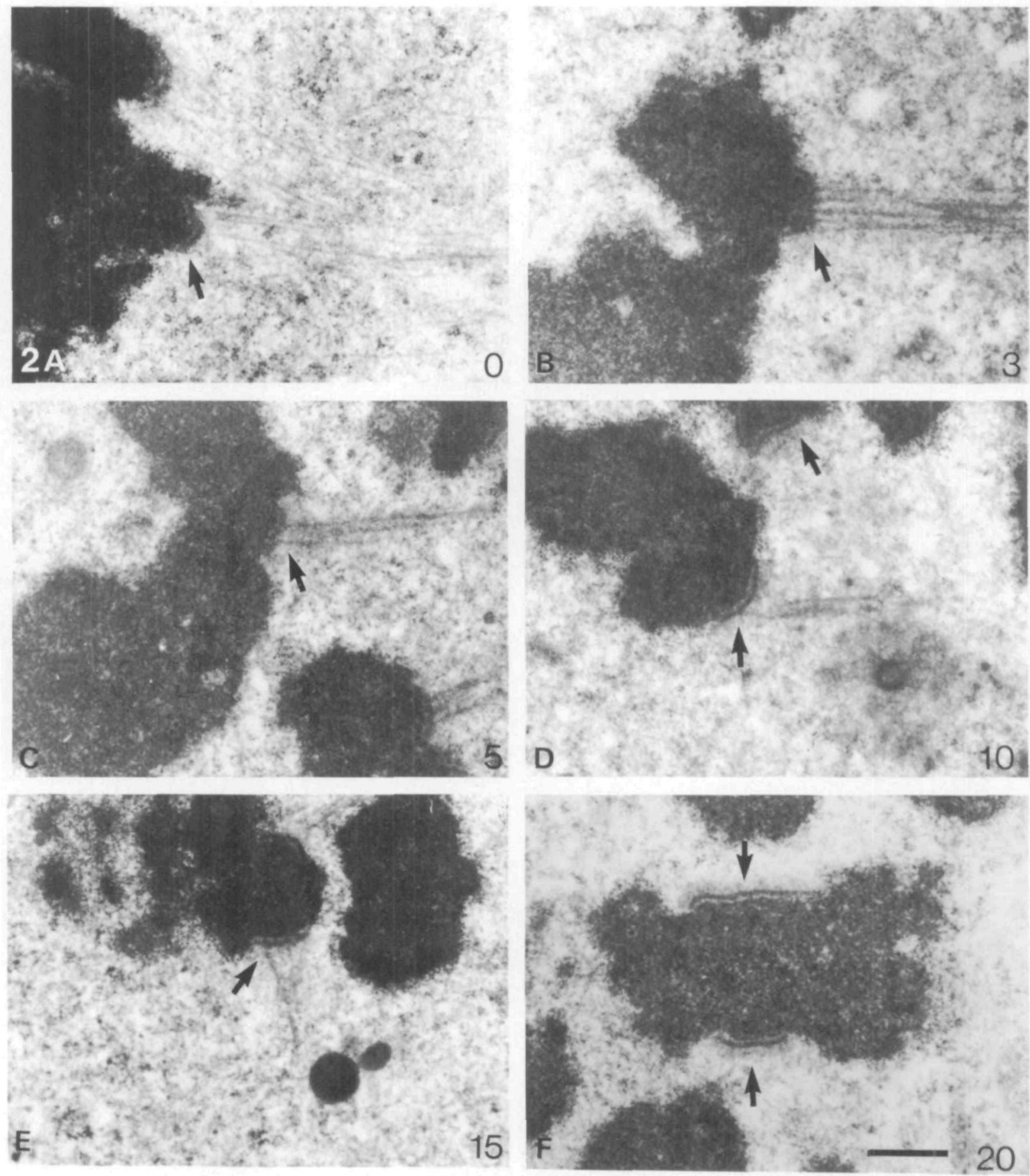

Fig. 2. Electron micrographs of representative sections through the kinetochore regions of cells fixed after incubation in nocodazole. The time of incubation (in min) in nocodazole is given in each frame. Kinetochores are denoted by arrows. Bar, $0.5 \mu \mathrm{m}$.

length of the kinetochore fibers from these and similar sets of serial sections through other cells fixed at different times after nocodazole treatment (Fig. 4). The average number of kinetochore microtubules was $24 \pm 5$ in control cells. After nocodazole addition, the mean number of kinetochore microtubules decreased as a first-order reaction with a half-life of $\sim 7.5 \mathrm{~min}$. The calculated half-life would be $\sim 30 \mathrm{~s}$ shorter without inclusion of the $15 \mathrm{~min}$ data point (Fig. 4), but since we have no reason to disregard the data from this seemingly anomalous cell, we have included this point in the calculation of the half-life of microtubule attachment to the kinetochore.

We also looked for structural changes in the kinetochore correlated with decreased numbers of microtubules. The kinetochore corona material (reviewed by Rieder, 1982; Brinkley et al. 1989) became very distinct, and appeared to radiate away from the kinetochore plate as the number of attached microtubules decreased (Figs 2F, 3C). No other obvious structural changes in the trilaminar appearance of the kinetochore were observed. 

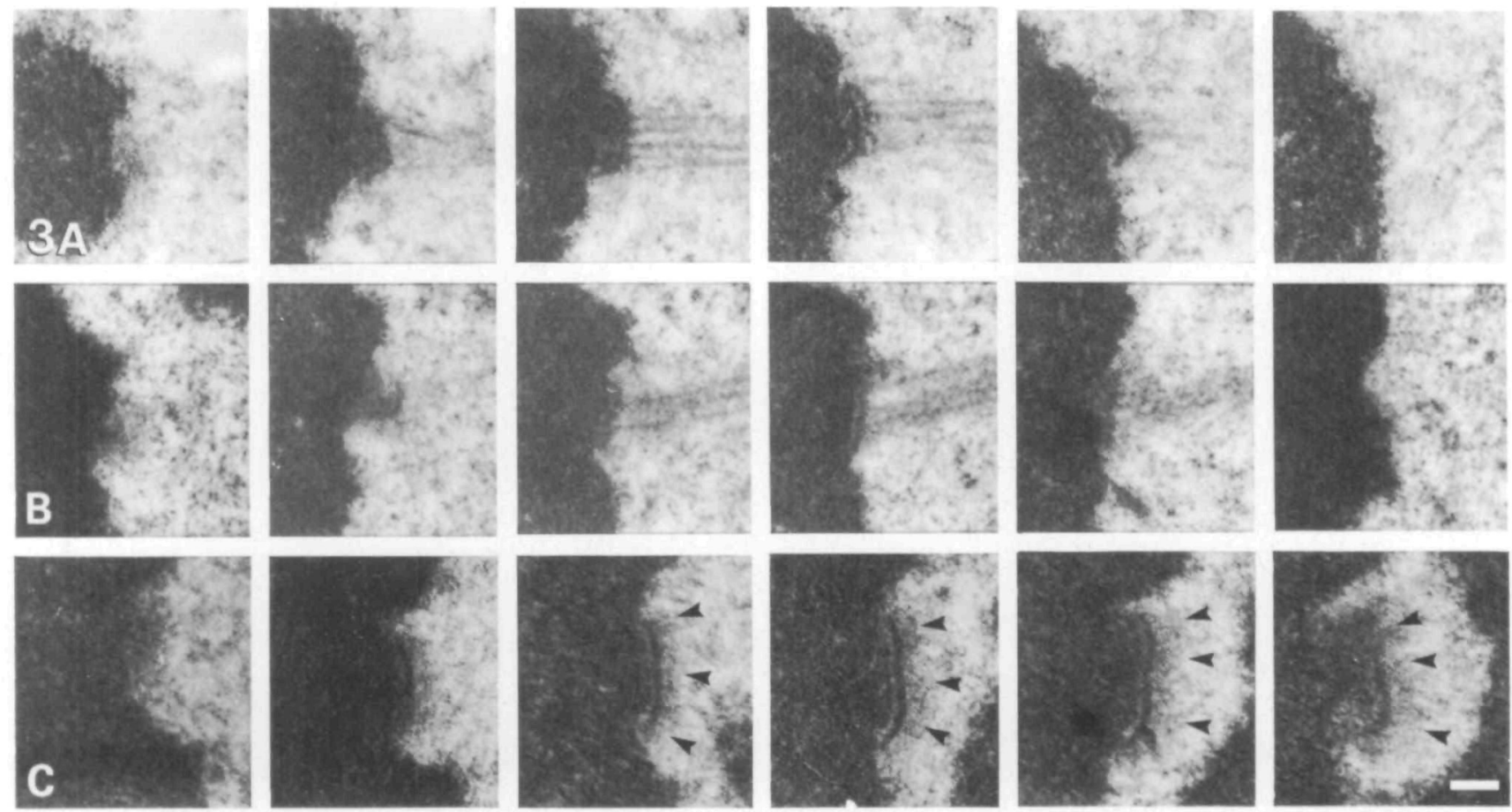

Fig. 3. Serial sections through kinetochores in cells fixed $3 \mathrm{~min}(\mathrm{~A}), 5 \mathrm{~min}(\mathrm{~B})$, and $20 \mathrm{~min}(\mathrm{C})$ after incubation in nocodazole. The corona material appears distinct and radiates away from the kinetochore after $20 \mathrm{~min}$ in nocodazole (arrowheads in $\mathrm{C}$ ). $\mathrm{Bar}, 0.25 \mu \mathrm{m}$.

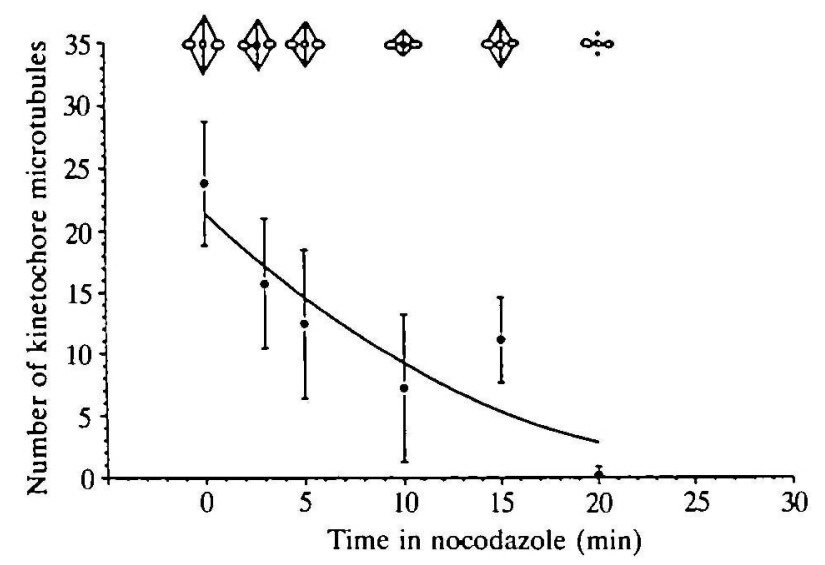

Fig. 4. Kinetics of kinetochore microtubule number loss after incubation in nocodazole. The number of kinetochore microtubules was determined from electron micrographs of serial sections as described in Materials and methods. Each data point represents the mean number of kinetochore microtubules per kinetochore \pm standard deviation. The relative length of the kinetochore fibers is shown along the upper portion of the graph directly above the corresponding data point.

\section{Discussion}

Our results showed that the average number of kinetochore microtubules in $\mathrm{PtK}_{1}$ metaphase cells at $23^{\circ} \mathrm{C}$ was $24 \pm 5$, approximately the same number as found previously for PtK kinetochores at $37^{\circ} \mathrm{C}$ (Brinkley and Cartwright, 1975; Roos, 1973) or $4^{\circ} \mathrm{C}$ (Rieder, 1981a). Because metaphase kinetochores of cells fixed at $37^{\circ} \mathrm{C}, 23^{\circ} \mathrm{C}$ and $4^{\circ} \mathrm{C}$ all have $\sim 24$ microtubules, this number is likely a measure of the average number of microtubule binding sites on $\mathrm{PtK}_{1}$ kinetochores.
Our results also showed that nocodazole at $34 \mu \mathrm{M}$ initiates three events in metaphase $\mathrm{PtK}_{1}$ cells: a rapid disappearance ( $<1 \mathrm{~min}$ ) of the majority of non-kinetochore polar and astral spindle microtubules; a shortening of persistent kinetochore fibers; and a slow loss of kinetochore microtubules characterized by a half-life of $\sim 7.5 \mathrm{~min}$. It is clear from these results that kinetochore microtubules are much more stable than the non-kinetochore polar microtubules, a result found previously for other microtubule-depolymerizing agents such as cooling, high hydrostatic pressure, calcium and colchicine-like drugs (Inoue, 1952, 1976; Brinkley and Cartwright, 1975; Lambert and Bajer, 1977; Salmon, 1975; Salmon and Segall, 1980; Salmon and Begg, 1980; Rieder, 1981a; Salmon et al. 1984).

The $\sim 7.5 \mathrm{~min}$ half-life for microtubule attachment to the kinetochore represents a minimum value for the stability of kinetochore microtubules, since two classes of mechanisms could generate the loss of kinetochore microtubules: (1) detachment induced by plus-end tubulin dissociation at the kinetochore without translocation to maintain microtubule attachment; or (2) detachment induced by tubulin dissociation at a site distal from the kinetochore (e.g. separation of minus-ends from the pole, with subsequent rapid minus-end depolymerization into the kinetochore). We cannot directly rule out either class of mechanism.

In addition to the mechanisms described above, it is also theoretically possible for the number of kinetochore microtubules to decrease by shortening at the kinetochore without any microtubule detachment. This decrease in the number of kinetochore microtubules could occur because not all kinetochore microtubules span the distance between the kinetochore and the pole (Rieder, 1981a). To determine whether this mechanism could account for the data, the theoretical numbers of kinetochore microtubules at positions closer to the pole (at positions approximating 
the amount of spindle shortening, see Fig. 4) were determined from the published length distribution of the coldstable kinetochore fiber (see Fig. 5, Rieder, 1981a). All microtubules were assumed to depolymerize from their plus-ends without detachment from the kinetochore. This mechanism could not account for our data, because it predicts very small changes in the number of microtubules attached to the kinetochore. For example, after $20 \mathrm{~min}$ in nocodazole this mechanism predicts that approximately $83 \%$ of the microtubules would remain attached to the kinetochore (based on Fig. 5 of Rieder, 1981a, the number of kinetochore microtubules would decrease from 29 to 24). In contrast, we found that the kinetochores were devoid of microtubules at this time point. Thus, the reduction in the number of kinetochore microtubules observed here cannot be accounted for solely by the shortening of kinetochore microtubules at the kinetochore without detachment of some microtubules during the shortening process.

These results have important implications for kinetochore regulation of microtubule turnover. According to the dynamic instability model of microtubule assembly dynamics (Mitchison and Kirschner, 1984; Kirschner and Mitchison, 1986), elongation of microtubules occurs by the addition of tubulin-GTP subunits to microtubule ends. Following dimer incorporation into an end, the GTP is hydrolyzed to GDP, producing a core of labile tubulin-GDP capped at the elongating end by newly added tubulin-GTP. When this 'tubulin-GTP cap' is lost, the microtubule end loses subunits rapidly and the microtubule rapidly shortens until it either disappears or is recapped. Since nocodazole-tubulin complex blocks microtubule elongation, both non-kinetochore and kinetochore microtubules are expected to lose their tubulin-GTP caps. The non-kinetochore microtubules rapidly disappear because their free plus-ends rapidly shorten back to the centrosome. The differential stability of the kinetochore microtubules is probably due to the attachment of their plusends to the kinetochore (Salmon et al. 1976; Mitchison and Kirschner, 1985), since other experiments have shown that kinetochore microtubules rapidly disappear upon detachment from the kinetochore (Nicklas and Kubai, 1985; Nicklas et al. 1982) and that microtubule plus-ends are stabilized in vitro by their attachment to kinetochores on isolated chromosomes (Mitchison and Kirschner, 1985). After nocodazole treatment, the rapid shortening expected for the tubulin-GDP ends of the microtubules attached to the kinetochores must be suppressed by the attachment sites within the kinetochore.

Although the kinetochore microtubules persist in nocodazole, they shorten as the poles move closer to the kinetochores at the metaphase plate. The site of tubulin subunit dissociation from kinetochore microtubules under these conditions (nocodazole-induced fiber shortening) has been determined by marking a position on the kinetochore fibers using fluorescence photobleaching. These experiments indicated that the majority of shortening occurred proximal to the kinetochore (Cassimeris and Salmon, 1988; Centonze and Borisy, 1988). Although some shortening may also occur proximal to the pole (Cassimeris and Salmon, 1988; Centonze and Borisy, 1988; see also, Mitchison, 1989), these results suggest that tubulin subunits dissociate predominately from a site proximal to the kinetochore as kinetochore microtubules shorten in nocodazole. During poleward movement of chromosomes in anaphase, tubulin subunits also dissociate from kinetochore microtubules from a site proximal to the kinetochore (Mitchison, 1986; Gorbsky et al. 1988). Microtubule de- polymerization at the kinetochore implies that the microtubule attachment site of the kinetochore can translocate polewards along the surface of the microtubule as tubulin subunits dissociate from microtubule plus-ends (Mitchison, 1988).

The $\sim 7.5 \mathrm{~min}$ half-life for microtubule attachment to kinetochores in nocodazole-treated cells is approximately twice as short as the $17 \mathrm{~min}$ half-time measured in vitro for the attachment of the plus-ends of taxol-stabilized microtubules to kinetochores on isolated chromosomes (Huitorel and Kirschner, 1988). This comparison suggests that the value of $7.5 \mathrm{~min}$ may represent the in vivo probability of microtubule depolymerization out of the microtubule attachment site under conditions where microtubule assembly is inhibited. Tubulin dissociation without concurrent poleward translocation of the kinetochore would produce detachment of plus-ends, which then could rapidly shorten and disappear like the free-ends of the polar, nonkinetochore microtubules. Conversely, under microtubule assembly conditions (i.e. conditions in an untreated cell), the life-time of a kinetochore microtubule could be longer than the $\sim 7.5$ min half-life measured here.

The relatively long life-time of a kinetochore microtubule determined here has important implications for the mechanism of tubulin subunit incorporation and dissociation from kinetochore microtubules as kinetochore fibers change lengths during prometaphase and metaphase. Kinetochore fibers change length during prometaphase as chromosomes congregess to the metaphase plate. During metaphase, kinetochore fibers change length as chromosomes oscillate back and forth between the spindle poles over distances of several micrometers from the spindle equator for periods of 1-2 $\mathrm{min}$ in each direction (Salmon, 1989; D. Wise, L. Cassimeris, P. Wadsworth, C.L. Rieder and E.D. Salmon, unpublished data). Thus, the lifetime of a kinetochore microtubule measured here is at least twice as long as the typical chromosome oscillation period observed during metaphase. This is consistent with the idea that tubulin subunits can incorporate into, and dissociate from, elongating and shortening kinetochore microtubules, respectively, without detachment occurring.

We previously examined microtubule turnover in prometaphase-metaphase $\mathrm{PtK}_{1}$ spindles at $23^{\circ} \mathrm{C}$ using FRAP measurements (Cassimeris et al. 1988). The FRAP results indicated that $70 \%$ of the microtubules within the spindle at this temperature were dynamic and turned over with a half-time of $\sim 77 \mathrm{~s}$, while approximately $30 \%$ of the microtubules turned over at a much slower rate. In this preliminary report, we assumed that the majority of microtubules present at this temperature were kinetochore microtubules on the basis of the distinct appearance of kinetochore fibers in immunofluorescence micrographs such as those seen in Fig. 1A. Given this assumption, we suggested that the majority of kinetochore microtubules could be binding and releasing from the kinetochores at rates similar to the life-times of the polar non-kinetochore microtubules. This idea was initially proposed by McIntosh and Vigers (1987), based on FRAP measurements of $\mathrm{PtK}_{1}$ spindles at $37^{\circ} \mathrm{C}$. However, our subsequent ultrastructual analysis of the microtubule composition of $\mathrm{PtK}_{1}$ spindles at $23^{\circ} \mathrm{C}$ revealed that kinetochore microtubules represented only $30 \%$ of the microtubules within the spindle (D. Wise, L. Cassimeris, P. Wadsworth, C.L. Rieder and E.D. Salmon, unpublished data). This percentage $(30 \%)$ is identical to the percentage of microtubules within the spindle that turn over slowly as measured in our FRAP 
studies. Thus, our initial suggestion was wrong. At $23^{\circ} \mathrm{C}$, the life-time of kinetochore microtubules is much longer than the non-kinetochore polar microtubules as measured by the nocodazole studies reported here, the tubulin incorporation studies of Mitchison et al. (1986) as well as our FRAP experiments (Wadsworth and Salmon, 1986).

Recently, Gorbsky and Borisy (1989) examined kinetochore microtubule stability using FRAP techniques to mark kinetochore fiber microtubules in metaphase LLCPK cells at $37^{\circ} \mathrm{C}$. They estimated a half-time for turnover of microtubules within kinetochore fibers of about $70 \mathrm{~s}$ for $70 \%$ of the microtubules; $30 \%$ were relatively stable. A temperature-dependent turnover rate may account for the differences in microtubule stability observed by Gorbsky and Borisy (1989) and the results presented here. However, their FRAP numbers are strikingly similar to our previous FRAP results for $\mathrm{PtK}_{1}$ cells at $23^{\circ} \mathrm{C}$ (Cassimeris et al. 1988). As discussed above, the $30 \%$ photobleached fluorescence that did not recover over several minutes may represent microtubules attached to the kinetochore, while the $70 \%$ fluorescence recovery during the first minute after bleaching may correspond to the dynamics of nonkinetochore microtubules that may have remained clustered with the kinetochore microtubules during the lysis procedures used in their experiments.

We also observed the appearance of fuzzy, filamentous corona material on the surface of the kinetochore regions previously occupied by kinetochore microtubules (Figs 2 and 3 ). The coronal filaments were not apparent in regions of the kinetochore with attached microtubules (Fig. 2). If the corona filaments were stretched out and tightly associated with the microtubule walls in this latter case, the corona filaments might not be apparent because their contrast could be lost in the contrast of the microtubule walls. Almost nothing is known about the corona filaments (Rieder, 1982; Brinkley et al. 1989), but recent observations indicate they could be involved in poleward kinetochore translocation along the walls of microtubules (Rieder and Alexander, 1990). If sites on the corona filaments were also responsible for the attachment of microtubules to the kinetochore, the corona filaments could provide the structural basis for the ability of the kinetochore attachment site to couple translocation to the growth and shortening of microtubules without detachment occurring.

In summary, the results reported here show that microtubule attachment to kinetochore persists during the shortening of kinetochore fibers induced by inhibiting microtubule assembly with nocodazole. Detachment occurs slowly in comparison to the dynamics of non-kinetochore microtubule assembly and the durations of chromosome movement during metaphase and anaphase. Although infrequent, detachment is probably important for the events of chromosome re-orientation that are involved in sister (mitosis) and homolog (meiosis) kinetochores becoming attached to polar microtubules from opposite poles. Attachment of a kinetochore to microtubules from both poles inhibits chromosome segregation and results in aneuploidy (Nicklas, 1985).

Thenks to Drs Nancy Pryer, Richard Walker, Pat Wadsworth and Sally Zigmond for many helpful discussions. Thanks also to Brenda Bourns and Vicki Petrie for technical assistance and to Susan Whitfield for help with photography. L.C. also thanks Dr Samsonoff, the members of the Rieder bunch (Wadsworth Center) and Wilman Hanton (UNC) for help with electron microscopy. Supported by NIH GM24364 (to E.D.S.), NIH GMS 40198 (to
C.L.R.) and NIH PHS 01219 (to support the Wadsworth Center's Biological Microscopy Facility).

\section{References}

Brinklegy, B. R. AND CARTwright, J. (1975). Cold-labile and cold-gtable microtubules in the mitotic apindle of mammalian cells. Ann. N.Y. Acad. Scl. 253, 428-439.

Brinkley, B R, Valdivia, M. M., Tousson, A. and Balczon, R. D. (1989) The kinetochore: Structure and molecular organization In Mutosis: Mechanisms and Molecules (ed. J. S. Hyams and B. R. Brinkley), pp. 77-118. New York, London: Academic Press.

Cassimeris, L., Inoue, S. AND Salmon, E D (1988). Microtubule dynamics in the chromosomal spindle fiber: Analysis by fluorescence and high-resolution polarization microscopy. Cell Motal. Cytoskel. 10, 185-196.

Cassimeris, L. and Saladon, E. D. (1988). During prometaphase kinetochore microtubules shorten by loss of subunits at the kinetochore J. Cell Biol. 107, 239a.

Cassimeris, L. U., Wadsworth, P. and Salmon, E. D. (1986). Dynamice of microtubule depolymerization in monocytes. J. Cell Btol. 102, $2023-2032$

Centonze, V. E. And Borisy, G. G. (1988). Microtubule dynamics during anaphase-like movements induced at metaphase. J. Cell Btol. 107, $240 a$.

Geuens, G., Hild, A. M., Levilulers, N., Adoutte, A. AND DeBrabander, M. (1989). Microtubule dynamics investigated by microinjection of Paramecium axonemal tubulin: Lack of nucleation but proximal assembly of microtubules at the kinetochore during prometaphase. J. Cell Biol. 108, 939-953.

GorBsky, G. J. AND Bonsy, G. G. (1989) Microtubules of the kinetochore fiber turn over in metaphase but not in anaphase. J. Cell Biol. 109, $653-662$.

Gorbsky, G J , Sammak, P. J. and Borisy, G. G. (1988). Microtubule dynamics and chromosome motion visualized in living anaphase cells J. Cell Biol. 106, 1185-1192

HogakKe, J., VAN NiJEN, G and DeBrabander, M. (1976). interaction of oncodazole (R17934), a new antitumoral drug, with rat brain tubulin. Brochem. biophys. Res. Commun. 69, 319-324.

HuITORRL, P. AND KIrschner, M W (1988). The polarity and stability of microtubule capture by the kinetochore. J. Cell Biol. 106, 151-159.

INoue, S. (1952). The effect of colchicine on the microscopic and submicroscopic structure of the mitotic spindle. Expl Cell Res. Suppl. 2, $305-318$.

INOUE, $S$ (1976). Chromosome movement by reversible assembly of microtubules. In Cell Motılity. Cold Spring Harbor Conferences on Cell Proliferation, vol. 3 (ed. R. Goldman, T. Pollard and J. Rosenbaum), pp 1317-1328. Cold Spring Harbor Laboratory, Cold Spring Harbor, NY. Inoue, S. (1981). Cell division and the mitotic spindle. J. Cell Biol. 91, 131s-1478.

Kirschner, M AND Mitchison, T. (1986). Beyond self-assembly: From microtubules to morphogenesis. Cell 45, 329-342.

Koshiand, D. E., Mitchison, T. J. AND KirschnsR, M. W. (1988),

Polewards chromosome movement driven by microtubule depolymerization in vutro. Nature 331, 499-504

LAMBERT, A.-M. AND BAJBR, A. S. (1977). Microtubule distribution and reversible arrest of chromosome movements induced by low temperature. Cytobiologie 15, 1-23.

LEE, C , FigLD, D. J. AND LEE, L. L. Y (1980) Effects of nocodazole on structures of calf brain tubulin. Biochemistry 18, 6209-6216.

Margolis, R. L and WIL8ON, L. (1977) Addition of colchicine-tubulin complex to microtubule ends. The mechanism of substoichiometric colchicine polsening. Proc. natn. Acad Scl. U.S.A. 74, 3466-3470.

McIntosh, J. R. ANd Koonce, M. P. (1989). Mitosis. Science 246, 622-628.

McIntosh, J. R. AND VigBrs, G. P A. (1987). Microtubule dynamics in the mitotic spindle. In Proceedings of the 45th Annual Meeting of the Electron Microscopy Socuety of Amerzca (ed. G. W. Bailey), pp. 794-797. San Francisco, CA. San Francisco Prese.

Mrtchison, T (1988). Microtubule dynamics and kinetochore function in mitosis. A. Rev. Cell Biol. 4, 527-549.

Mrtchison, T. (1989). Polewards microtubule flux in the mitotic spindle: Evidence from photoactivation of fluorescence. J. Cell Biol. 109, $637-652$.

Mrtchison, T., Evang, L., Schulzz, E. And Kirschner, M (1986). Sites of microtubule assembly and disassembly in the mitotic spindle. Cell 45, 515-627.

Mitchison, T. AND Kirschner, M. (1984). Dynamic instability of microtubule growth. Nature 312, 237-242

Mitchison, T. J. and Kinschner, M. W. (1985). Properties of the kinetochore in vutro. II. Microtubule capture and ATP-dependent translocation. J. Cell Bıol. 101, 766-777. 
NICKLAs, R. B. (1985). Mitosis in eukaryotic cells: An overview of chromosome distribution. In Aneuploidy. Etrology and Mechantsms (ed. V. L. Dellarco, P. E. Voytek, A. Hollaender), pp. 183-195 New York: Plenum Press

NickLAs, R. B. (1988) Chromosomes and kinetochores do more in mitosis than previously thought In Chromosome Structure and Function: The Impact of New Concepts (ed. J. P. Gustafson and R. Appels), pp. 53-74. New York: Plenum Press.

Nicklas, R. B. And KubaI, D. F. (1985). Microtubules, chromosome movement, and reorientation after chromosomes are detached from the spindle by micromanipulation. Chromosoma 92, 313-324.

Nicklas, R. B , Kubai, D. F. and Hays, T. S. (1982). Spindle microtubules and their mechanical assaciations after micromanipulation in anaphase. J. Cell Brol. 95, 91-104.

RigDer, C. L. (1981a). The structure of the cold-stable kinetochore fiber in metaphase $\mathrm{PtK}_{1}$ cells. Chromosoma 84, 145-158.

RIEDER, C. L. (1981b). Effect of hypothermia $\left(20-25^{\circ} \mathrm{C}\right)$ on mitosis in $\mathrm{PtK}_{1}$ celle. Cell Biol. Int. Rep. 5, 563-573.

RIEDER, C. L. (1982) The formation, structure, and composition of the mammalian kinetochore and kinetochore fiber. Int. Rev. Cytol. 78 $1-57$.

Rigder, C. L. ANd AleXander, S. P. (1990). Kinetochores are transported poleward along a single astral microtubule during chromosome attachment to the spindle in newt lung cell $J$ Cell Biol. 110, 81-95.

Roos, U.-P. (1973). Light and electron microscopy of rat kangaroo cells in mitosis. Chromosoma 41, 195-220.

Salmon, E D. (1975). Spindle microtubules. Thermodynamics of tn vivo gsembly and role in chromosome movement. Ann. $N Y$ Acad Sci. 253, 383-406.

SAlmon, E. D. (1989). Microtubule dynamics and chromosome movement. In Mitosus: Molecules and Mechanisms (ed. J. S. Hyams and B. R. Brinkley). pp. 119-181. New York, London: Academic Press.

SAlMon, E. D. AND BeGG, D. A. (1980). Functional implications of coldstable microtubules in kinetochore fibers of insect spermatocytes during anaphase. J. Cell Biol. 85, 853-865.

Salmon, E. D , Goode, D., Maugel, T. K. and Bonar, D. B. (1976). Pressure-induced depolymerization of spindle microtubules. III. Differential stability in HeLa cells. J. Cell Btol. 69, 443-454

Salmon, E. D., McKeEl, M. and Hays, T. (1984). Rapid rate of tubulin dissociation from microtubules in the mitotic spindle in vivo measured by blocking polymerization with colchicine. J. Cell Biol. 99, 1066-1075.

Salmon, E. D. AND Segall, R. R. (1980). Calcium-labile mitotic spindles isolated from sea-urchin egg8 (Lytechinus variegatus), J. Cell Biol. 86 $355-365$.

WaDsworth, P. AND Salmon, E. D. (1986). Analysis of the treadmilling model during metaphase of mitosis using fluorescence redistribution after photobleaching. J. Cell Biol. 102, 1032-1038.

WITT, P. L., RIs, H. AND BonIsY, G. G. (1981). Structure of kinetochore fibers: Microtubule continuity and inter-microtubule bridges. Chromosoma 83, 523-540.

(Recelved 18 December 1989 - Accepted 15 February 1990) 
